\title{
Quantization of Crack Speeds in Dynamic Fracture of Silicon: Multiparadigm ReaxFF Modeling
}

Harvey Tang ${ }^{1}$, Janet Rye ${ }^{2}$, Markus J. Buehler ${ }^{3}$, Adri van Duin ${ }^{4}$, and William A. Goddard III ${ }^{4}$

'Department of Aeronautics and Astronautics, Massachusetts Institute of Technology, Cambridge, MA, 02139

${ }^{2}$ Materials Science and Engrg., Massachusetts Institute of Technology, Cambridge, MA, 02139

${ }^{3}$ Civil and Environmental Engrg., Massachusetts Institute of Technology, Cambridge, MA, 02139

${ }^{4}$ Division of Chemistry and Chemical Engineering, California Institute of Technology, Pasadena, CA, 91125

Corresponding author, electronic address: mbuehler@MIT.EDU

\section{ABSTRACT}

We report a study of dynamic cracking in a silicon single crystal in which the ReaxFF reactive force field is used for about 3,000 atoms near the crack tip while the other 100,000 atoms of the model system are described with a simple nonreactive force field. The ReaxFF is completely derived from quantum mechanical calculations of simple silicon systems without any empirical parameters. This model has been successfully used to study crack dynamics in silicon, capable of reproducing key experimental results such as orientation dependence of crack dynamics (Buehler et al., Phys. Rev. Lett., 2006). In this article, we focus on crack speeds as a function of loading and crack propagation mechanisms. We find that the steady state crack speed does not increase continuously with applied load, but instead jumps to a finite value immediately after the critical load, followed by a regime of slow increase. Our results quantitatively reproduce experimental observations of crack speeds during fracture in silicon along the (111) planes, confirming the existence of lattice trapping effects. We observe similar effects in the (110) crack direction.

\section{INTRODUCTION}

Brittle fracture is characterized by breaking of atomic bonds leading to formation of two

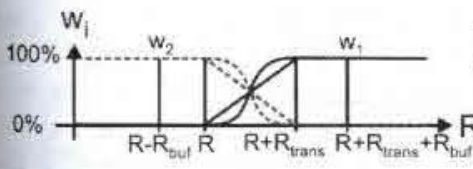

\section{ReaxfF}

(a)

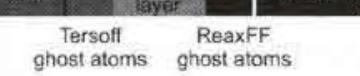

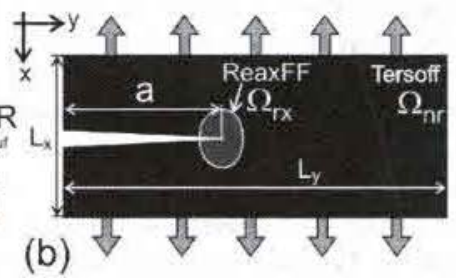

Figure 1: Subplot (a): The interpolation method for defining a mixed Hamiltonian in the transition region between two different paradigms, as implemented in the CMDF framework.

As an alternative to the linear interpolation, we have also implemented smooth interpolation function based on a sinusoidal function. This enables using slightly smaller handshake regions thus increasing the computational efficiency. Subplot (b): Geometry used for simulating mode I fracture in silicon. The (110) crack surface system contains 28,800 atoms with $L_{\gamma}=230 \AA$, and $L_{\gamma} \approx 460 \AA$. The (111) crack surface system contains 86,400 atoms with $\mathrm{L}_{2}=400 \AA$, and $\mathrm{L}_{\gamma}=1130 \AA$. At the tip of the crack, up to 3,000 atoms are modeled with ReaxFF. 
new materials surfaces. Most existing atomistic models of fracture assume an empirical

Crack Speed vs: Loading (110)

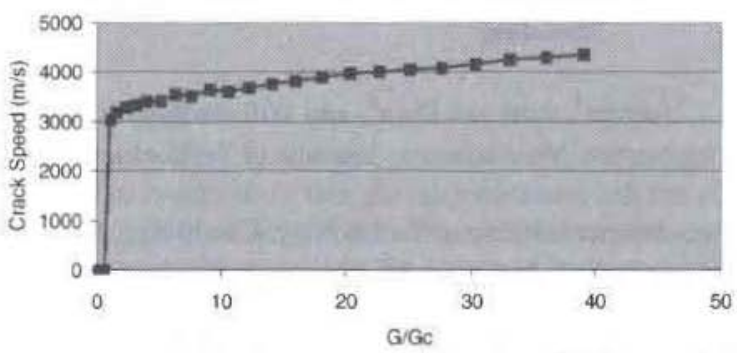

Figure 2: Crack speed as a function of reduced load, for a crack in the (110) orientation. For small loads $\left(G / G_{C}<1\right)$, the crack speed is zero. Above $G / G_{C}>1$ we observe a finite crack speed, with a sudden jump to approximately $3,000 \mathrm{~m} / \mathrm{s}$. Then the crack speed displays a steady, linearly increasing, trend with strain load.

The average limiting crack speed is measured while the crack travels in the region $200 \AA<y<400 \AA$ (sufficiently far away from the boundary). The crack speed at large loading approaches approximately $4,200 \mathrm{~m} / \mathrm{s}$.

relationship between bond stretch and force. However, breaking of bonds in real materials is an extremely complicated process that could previously only be captured with sufficient accuracy only by using quantum mechanical (QM) methods, which are limited to $\sim 100$ atoms. Here we present a new theoretical concept based on building a multi-scale simulation model completely derived from QM principles, while being computationally efficient and capable of treating thousands of atoms with QM accuracy. Fracture of silicon has received tremendous attention due to its complexity of bond breaking and due to interesting failure dynamics observed experimentally [1-5].

Atomistic modeling fracture of silicon has been the subject of several studies using empirical force fields [5-9]. In contrast to many metals, a proper description of fracture in silicon has proved to be far more difficult, requiring a more accurate treatment of the atomic interactions. There have been several previous attempts to describe fracture of silicon using atomistic methods. Early attempts to model fracture in Si used Tersoff's classical potential [10] and similar formulations such as the Stillinger-Weber [11], the MEAM method [9] or the EDIP potential [8] failed due to an incorrect description of the bond breaking process. It has become clear that in order to obtain an accurate description of the dynamics of fracture of materials, the accuracy of QM for atoms near the propagating crack tip is necessary to describe the very large potentially plastic strains as chemical bonds are broken while coupling this to the more gradual near elastic strains over much larger regions. This has been successfully demonstrated in earliet work [6, 12].

Here we present the results for an alternative approach utilizing the ReaxFF reactive force field developed to reproduce the barriers and structures for reactive processes from QM. but at a computational cost many orders of magnitude smaller. We use a hybrid simulation technique in which the ReaxFF reactive potential for silicon [13, 14] is used for a modest region of a few thousand atoms close to the crack tip while a computationally inexpensive but nonreactive Tersoff potential is used to describe the other 100,000 more distant atoms needed to include their elastic constraints on the propagating tip. Tersoff-type potential and ReaxFF lead to similar materials behavior (equation of state) for small strains, but deviate strongly at lagge strains. The fact that both descriptions overlap for small strains enable a smooth handshake between the two methods. Details of this method are described elsewhere [15]. 


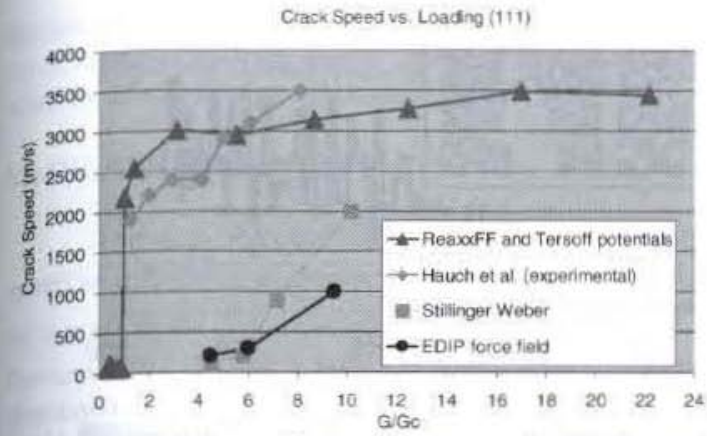

Figure 3: Crack speed as a function of reduced load, for a crack in the (111) orientation. For small loads $\left(G / G_{C}<1\right)$, the crack speed is zero. Above $G / G_{C}>1$ we observe a finite crack speed, with a sudden jump to approximately $2,000 \mathrm{~m} / \mathrm{s}$. Then the crack speed displays a steady, linearly increasing, trend with strain load, approaching approximately $3,500 \mathrm{~m} / \mathrm{s}$.

The average limiting crack speed is measured while the crack travels in the region $250 \AA<y<400 \AA$ (sufficiently far away from the boundaries). At the intermediate values around $G / G_{C} \approx 1$, the crack does not propagate through the entire slab. When stable crack propagation is observed, the average crack speed approaches the initial velocity of approximately $2,000 \mathrm{~m} / \mathrm{s}$. Our results show a quantitative correlation with the experimental results obtained by Hauch and coworkers [12] (representative data plotted in the figure). The plot also contains data obtained with the Stillinger-Weber force field and the EDIP force field (data taken from [12]); these models fail to reproduce the experimental behavior.

\section{COMPUTATIONAL METHOD: HYBRID ReaxFF-Tersoff MODEL}

The Computational Materials Design Facility (CMDF) is a Python [16] based simulation framework allowing multi-paradigm multi-scale simulations of complex materials phenomena operating on disparate length- and time scales. Individual computational engines are wrapped using the "Simplified Wrapper and Interface Generator" (SWIG) for rapid integration of lowlevel codes with scripting languages. The CMDF framework enables the combination of ReaxFF to capture the QM description of reactions with classical non-reactive potentials to describe nonreacting regions providing the means for describing many complex materials failure processes as reported herein. This is the main focus of this paper.

To simulate the atoms around the crack tip we employ ReaxFF [13]. As described in [14], this potential has been tested against QM for a wide range of processes [17], including Si-Si bond breaking in $\mathrm{H} 33 \mathrm{Si}-\mathrm{SiH} 3$ and $\mathrm{Si}=\mathrm{Si}$ bond breaking in $\mathrm{H}_{2} \mathrm{Si}=\mathrm{SiH}_{2}$, equations of state for 4coordinate silicon (diamond-configurations) and 6-coordinate silicon phases $\beta$-tin), and simple cubic crystal. This force field is also capable of treating interactions of $\mathrm{Si}$ with $\mathrm{O}$ and $\mathrm{H}$. We recently added the stability and equation of state of a 5-coordinate Si-condensed phase to the Si training set, and we re-evaluated the $\mathrm{Si}-\mathrm{Si}$ bond and $\mathrm{Si}$-Si-Si angle parameters to improve the fit to the energy and equation of state of this phase. All parameters are completely derived from QM calculations. Because of the complexity of the mathematical expressions describing the partial bond orders, energies, and charges, ReaxFF is 1-2 orders of magnitudes more expensive than the Tersoff potential but several orders of magnitude faster than QM.

We use a simple general approach to describe the transition region between the two paradigms ReaxFF and Tersoff. The role of the handshake regions is to ensure that the correct boundary conditions are applied to each side, and that each side senses proper continuation in distribution or density and forces. Figure l(a) illustrates the approach. The transition region is described by two parameters, $R_{\text {tras }}$ for the width of the transition region, and $R_{\text {pur }}$ for the width of the ghost atom region. In bridging such distinct computational engines we find it useful to use 


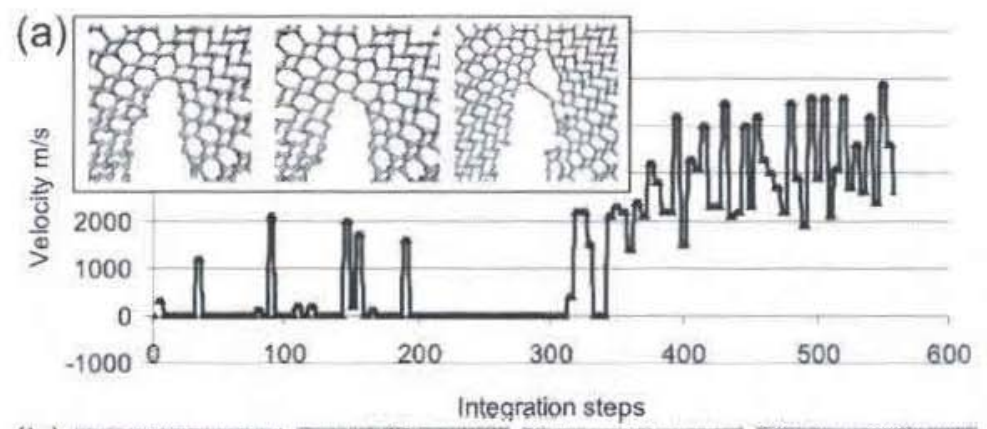

(b)
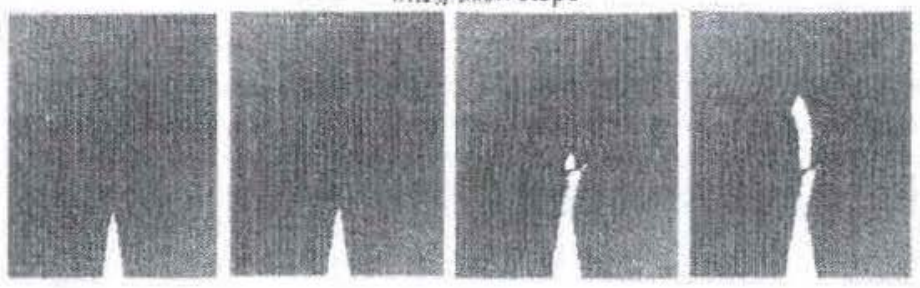

Figure 4: Subplot (a): Crack speed as a function of iterations. The inlays show a few snapshots of bond breaking processes during crack initiation. We observe that the crack does not start to propagate until a critical loading time, after which the crack speed jumps to around $2,000 \mathrm{~m} / \mathrm{s}$. This result agrees with the data shown in Figure 2 and 3, suggesting that crack propagation is not possible for speeds below a critical speed. Preliminary analysis suggests that this behavior is due to atomic rearrangements at the tip of the crack, leading to formation of a 7-5 membered Si-ring. Subplot (b): Snapshots of crack extension during the simulation. After a short period of straight crack extension, the crack starts to change direction, creating a rough crack surface.

spatially varying weights $w_{i}$ to determine the weighting of the force and energy contribution from different simulation engines. Every computational engine $i$ has a specific weight $w_{i}$ associated with it. The $N$ weights always add up to one, as $\sum_{t=1, \ldots N} w_{i}=1$. The force on each atom is then given by the weighted sum of all force contributions of each method $F=\sum_{i=1, N} w_{i} F_{i}$, Different simulation regimes are coupled to one another by smoothly interpolating between different engines by using smoothly varying weighting functions. The width of the transition region $R_{\text {trans }}$ depends on the nature of the system, but it should generally be larger than the typical atomic distance in a lattice or in an organic molecule (here we choose $R_{\text {trans }}=6 \AA$ for the (110) orientation and $R_{\tan }=5 \AA$ for the (111) orientation). The width of the buffer layer is $R_{M}$ $=5 \AA$ for both orientations. The size of the reactive region is based on the strain energy density of each atom. All atoms around the crack tip are embedded in a cylindrical reactive region of 15 $\AA$ and $30 \AA$, for the $(110)$ and (111) systems, respectively. We update the calculation regimes every 20 integration steps. Figure 1(b) depicts our atomistic model. We consider a perfect crystal with an initial crack of length $a$ serving as the failure initiation point. We strain the slab with strain in mode I prior to simulation as done in [18]. The boundaries are held fixed during 
the simulation so that the stress in the material can only be relieved by crack propagation. The crack starts to nucleate shortly after the simulation is started. The crack speed is averaged in a window of steady-state crack motion.

\section{SIMULATION RESULTS}

The main objective of this paper is to perform a systematic study of the steady-state crack speed as a function of the applied load. Figure 2 plots the crack speed as a function of $G / G_{C}$. for a crack in the (110) orientation. Note that $G_{c}$ is the critical energy release rate at the onset of fracture. We observe a lattice-trapping effect, similar as seen in experimental studies [4] and discussed in computational modeling recently [12]. Figure 3 plots the results obtained for the (111) orientation. This is the identical orientation as used in experiment and computational studies. Our hybrid model is capable of reproducing the experimental results quite accurately, showing some quantitative agreement. In the studies shown in Figures 2 and 3, the load applied to the system is held constant during the simulation.

Figure 4 shows the results for slowly increasing load (strain rate $0.0005 \%$ per integration step). Figure 4(a) shows the crack speed as a function of iteration number, including some snapshots of crack tip mechanisms and bond breaking events. Figure 4(b) shows some snapshot of the system as the crack propagates.

\section{DISCUSSION AND CONCLUSION}

We have presented a new numerical method integrating the ReaxFF and Tersoff force fields to allow a physics based description of the fracture mechanics of silicon. Our new scheme represents a new multi-scale approach of coupling the QM scale of chemistry and bond breaking and formation with the scale of mechanics of materials. Our method is completely based on firstprinciples, with no empirical parameters used for fitting of ReaxFF for silicon. Our studies suggest that there exist lattice trapping effects that lead to a discretization of accessible crack speeds in silicon, clearly corroborating earlier experimental results (Figures 2 and 3 ). In contrast to previous attempts of modeling this experimental phenomenon with empirical potentials such as EDIP or Stillinger-Weber, our new hybrid model reproduces experimental results, at a fraction of the computational cost of QM based methods. It is remarkable that our model is capable of even reproducing experimental results quantitatively (see Figure 4), without any parameter fitting. Our theoretical results further provide some insight into the crack-tip mechanisms during fracture initiation, indicating formation of a 5-7 membered Si ring (see inlay, Figure 4).

Since ReaxFF is capable of describing a wide heterogeneous range of materials, our approach provides a practical means to studying the coupling of complex chemical reactions to mechanical properties. Our hybrid method could enable studies of stress corrosion processes and other degradation and aging mechanisms.

\section{ACKNOWLEDGEMENTS}

HT and JR acknowledge support from MTT's UROP program. MJB acknowledges support from the MIT's CEE department. We thank Dr. C. Schwartz and S. Wax of DARPA for encouraging this development by funding first a special CMDF project and then the CMDF part 
of the DARPA-PROM project. Additional funding for this project was provided by DOE-ASC and NSF-ITR.

\section{REFERENCES}

1. Deegan, R.D., et al., Wavy and rough cracks in silicon. Phys. Rev. E, 2003. 67(6): p. 066209 .

2. Cramer, T., A. Wanner, and P. Gumbsch, Energy dissipation and path instabilities in dynamic fracture of silicon single crystals. Phys. Rev. Lett., 2000. 85: p. 788-791.

3. Cramer, T., A. Wanner, and P. Gumbsch, Crack Velocities during Dynamic Fracture of Glass and Single Crystalline Silicon. Phys. Status Solidi A, 1997. 164: p. R5.

4. Hauch, J.A., et al., Dynamic fracture in Single Crystal Silicon. Phys. Rev. Lett., 1999. 82: p. $3823-2826$.

5. Holland, D. and M. Marder, Ideal brittle fracture of silicon studied with molecular dynamics. Phys. Rev. Lett., 1998. 80(4): p. 746.

6. Abraham, F.F., et al., Spanning the length scales in dynamic simulation. Computers in Physics, 1998. 12(6): p. 538-546.

7. Bailey, N.P. and J.P. Sethna, Macroscopic measure of the cohesive length scale: Fracture of notched single-crystal silicon. Phys. Rev. B, 2003, 68(20): p. 205204.

8. Bazant, M.Z., E. Kaxiras, and J.F. Justo, Environment-Dependent Interatomic Potential for bulk silicon. Physical Review B-Condensed Matter, 1997. 56: p. 8542.

9. Swadener, J.G., M.I. Baskes, and M. Nastasi, Molecular Dynamics Simulation of Brittle Fracture in Silicon. Phys. Rev. Lett.. 2002. 89(8): p. 085503.

10. Tersoff, J., Empirical interatomic potentials for carbon, with applications to amorphous carbon. Phys. Rev. Lett., 1988, 61(25): p. 2879-2883.

11. Stillinger, F. and T.A. Weber, Computer-simulation of local order in condensed phases of silicon. Phys. Rev, B, 1985. 31(8): p. 5262-5271.

12. Bernstein, N. and D.W. Hess, Lattice trapping barriers to brittle fracture. Physical Review Letters, 2003.91(2).

13. Duin, A.C.T.v., et al., ReaxFF: A Reactive Force Field for Hydrocarbons. J. Phys, Chem. A, 2001. 105: p. 9396-9409.

14. Duin, A.C.T.v., et al., ReaxFF SiO: Reactive Force Field for Silicon and Silicon Oxide Systems. J. Phys. Chem. A, 2003. 107: p. 3803-3811.

15. Buehler, M.J., A.C.T.v. Duin, and W.A. Goddard, Multi-paradigm modeling of dynamical crack propagation in silicon using the ReaxFF reactive force field. Phys. Rev. Lett., 2006. 96(9): p. 095505.

16. Parker, S.G., C.R. Johnson, and D. Beazley, Computational steering software systems and strategies. IEEE Computational Science and Engineering, 1997. 4(4): p. 50-599.

17. Becke, A.D., Density-function thermochemistry. 3. The role of exact exchange. J. Chem. Phys., 1993. 98(7): p. 5648-5652.

18. Abraham, F.F., et al., Simulating materials failure by using up to one billion atoms and the world's fastest computer: Work-hardening. P. Natl. Acad. Sci. USA, 2002. 99(9): p. 5783-5787. 